\title{
Regarding "What Is the Ideal Core Number for Ultrasonography-Guided Thyroid Biopsy of Cytologically Inconclusive Nodules?"
}

W e read with great interest the article "What Is the Ideal Core Number for Ultrasonography-Guided Thyroid Biopsy of Cytologically Inconclusive Nodules?” by Hahn et al. ${ }^{1}$ In that study, the authors evaluated the correct number of core-needle specimens for patients with previously inconclusive fine-needle aspiration cytology (FNA) results and suggested that at least 2 core specimens containing intranodular and capsular tissue are necessary for a proper diagnosis. In their results, the diagnostic ability of 2 specimens was found to be higher than that of 1 specimen and was similar to that of 3 specimens. This concept is concordant with that of the recent core-needle biopsy (CNB) guidelines published by the Korean Society of Thyroid Radiology. ${ }^{2}$ Application of the results of Hahn et al $^{1}$ will help patients to avoid repeated FNAs or diagnostic surgery. Thanks to the valuable results in $\mathrm{CNB}$ skills and pathologic evaluation of core specimens. This study explained comprehensive aspects of the number of core specimens, and this concept had not been reported to date. The authors revealed several important aspects of core biopsy after previously inconclusive FNA results: 1) CNB achieved no nondiagnostic results; 2 ) the rate of inconclusive results was just $6.7 \%$ (4 of 60 ); 3 ) the final surgical pathology was well correlated with the core biopsy results; and 4) CNB procedures were tolerable in all patients. Thus, CNB is an effective and safe procedure for patients with previously inconclusive results, and these findings are concordant with those of previous CNB studies.

Although the study of Hahn et $\mathrm{al}^{1}$ obtained informative results, there are 3 points to be considered. The first is the relationship between the number of core specimens and the size of the specimen notch of the core device. Second, capsule information is necessary, especially for diagnosing follicular neoplasm; however, a core technique including the capsule is not recommended in all patients, especially those with suspected papillary thyroid cancers. Finally, to justify 2 core specimens, the complications of the 3 groups should be compared. Regarding the relationship between the core specimen number and the size of the specimen notch of the core device, a core device with a large specimen notch requires fewer core specimens. The authors used a 1.1-cm excursion core

http://dx.doi.org/10.3174/ajnr.A5231 device, which has a $0.7-\mathrm{cm}$ specimen notch. This obtains a core specimen of approximately $0.7 \mathrm{~cm}$ in length. A $1.6-\mathrm{cm}$ excursion core device can obtain a $1.2-\mathrm{cm}$ specimen length, whereas a $2-\mathrm{cm}$ excursion device can obtain a 1.6- $\mathrm{cm}$ specimen length. Therefore, 2 specimens obtained by a $1.1-\mathrm{cm}$ device are similar to 1 specimen obtained by a 1.6- or 2-cm core device. In addition, an 18-gauge core device can obtain larger-bore tissue than a 20- or 21-gauge core device. However, complications should be compared among devices with different lengths or thicknesses. Regarding capsule information, capsules can help to diagnose follicular neoplasms. Current CNB guidelines recommend that a nodule capsule be obtained during core biopsy. ${ }^{2}$ However, capsule information is unnecessary for the diagnosis of papillary thyroid cancers. Therefore, the use of an extra core biopsy should be restricted to certain types of thyroid nodules. Suh et $\mathrm{al}^{3}$ performed less than 2 core biopsy, but inconclusive results is not high in their factor analysis. Multiple biopsies can cause more complications; this issue should be considered during CNB procedures. To minimize complications, the operators performing CNB should understand the basic techniques and perithyroidal structures. ${ }^{4}$

In conclusion, Hahn et $\mathrm{al}^{1}$ have reported valuable results on the number of core specimens needed for patients with previously inconclusive FNA results in thyroid biopsy. Although these data are practically important, knowledge of the core device, the relationship between specimen notch and specimen length, the type of thyroid nodule, and possible complications is essential for operators to perform thyroid CNB.

Disclosures: Jung Hwan Baek—UNRELATED: Consultancy: RFA Company, ATARmed and RF Medical, Comments: since 2017, \$2700/year.

\section{REFERENCES}

1. Hahn SY, Shin JH, Oh YL. What is the ideal core number for ultrasonography-guided thyroid biopsy of cytologically inconclusive nodules? AJNR Am J Neuroradiol 2017;38:777-81 CrossRef Medline

2. Na DG, Baek JH, Jung SL, et al. Core needle biopsy of the thyroid: 2016 consensus statement and recommendations from Korean Society of Thyroid Radiology. Korean J Radiol 2017;18:217-37 CrossRef Medline

3. Suh $\mathrm{CH}, \mathrm{Baek} \mathrm{JH}$, Lee JH, et al. The role of core-needle biopsy as a 
first-line diagnostic tool for initially detected thyroid nodules. Thyroid 2016;26:395-403 CrossRef Medline

4. Ha EJ, Baek JH, Lee JH. Ultrasonography-based thyroidal and perithyroidal anatomy and its clinical significance. Korean J Radiol 2015; 16:749-66 CrossRef Medline

(1D) H.S. Park

(i).H. Baek

Department of Radiology and Research Institute of Radiology University of Ulsan College of Medicine
Asan Medical Center Seoul, Korea

(D) N.D. Gyu

Department of Radiology

GangNeung Asan Hospital

Seoul, Korea

Department of Radiology

Human Medical Imaging and Intervention Center

Seoul, Korea 